\title{
Formulation of Sodium Alginate Nanospheres Containing Amphotericin B for the Treatment of Systemic Candidiasis
}

\author{
Shanmugasundaram Sangeetha*, Dhandapani Nagasamy \\ Venkatesh, Rajendran Adhiyaman, Kumaraswamy Santhi, and \\ Bhojraj Suresh \\ Centre for Research and Post-graduate studies JSS College of Pharmacy Ooty-643 001. The Nilgiris, India.
}

\begin{abstract}
Purpose: The aim of this work was to formulate sodium alginate nanospheres of amphotericin $B$ by controlled gellification method and to evaluate the role of the nanospheres as a "passive carrier" in targeted antifungal therapy.

Methods: Sodium alginate nanospheres of amphotericin B were prepared by controlled gellification method, and the particle size analysis was carried out by scanning electron microscopy. The carrier capacity of sodium alginate was evaluated in terms of drug to polymer ratio. In vitro release study was carried out on all drug loaded nanospheres by the dialysis method. Release kinetics of drug from different drug loaded nanospheres was also determined. The in vivo antifungal efficacy of nanospheres bound drug vis-à-vis the free drug was evaluated in candidiasis- induced mice models.

Results: Preparation of nanospheres through controlled gellification method yielded particles with a size range of $419.6 \pm 0.28 \mathrm{~nm}$. Studies on drug to polymer ratio showed a linear relationship between concentration of drug and drug loading capacity. In vitro release kinetic study revealed that the release of drug from the nanospheres followed Fickian diffusion. In vivo studies showed that the nanospherebound drug produced a higher antifungal efficacy than the free drug.

Conclusion: The formulated sodium alginate nanospheres containing amphotericin $B$ was found to have better antifungal activity when compared to the free drug and also yielded sustained in vitro release.
\end{abstract}

Keywords: Nanospheres, sodium alginate, amphotericin B, controlled gellification method, in vitro \& in vivo release.

${ }^{*}$ Correspondence E-mail : sangeethamadhesh@rediffmail.com, Tel: +91-423-2443393. Fax: +91-423-2442937. 


\section{INTRODUCTION}

Infections due to fungi of the genera Candida and Aspergillus have become major nosocomial causes of morbidity and mortality in immunocompromised individuals. Their incidence has increased during the last three decades in parallel with the number of such patients and it is now a frequent complication ${ }^{1,2}$. More recently there has been a substantial increase in the frequency of candidaemia caused by species other than Candida albicans. ${ }^{3}$

Systemic candidiasis is associated with high mortality rate and prolonged hospitalization. ${ }^{4}$ The treatment options are extremely limited. Rapid treatment with amphotericin B or fluconazole is required to reduce the mortality observed in these patients. ${ }^{5}$ Amphotericin B is a very potent antifungal agent, but it has multiple severe toxic effects and about $36 \%$ rate of failure in the treatment of immunocompromised hosts. ${ }^{6}$ Thus several lipid formulations (Ambisome ${ }^{\circ}$, Amphocil $($, Abelcet $($ ) have been developed and commercialized. $^{7,8}$ Although they have been proven to reduce amphotericin B toxicity, their toxic effects and pharmacokinetic properties all differ and their use has been limited by high cost.

One of the suitable methods to overcome these problems could be association with biodegradable polymeric carriers such as nanoparticles. Nanoparticles may become one of the successful carriers by overcoming problems caused by infections that are refractory to conventional treatment and also widening the therapeutic margin of antibiotics currently used in clinical practice. ${ }^{9,10,11,12}$ Hence, the objective of the work was to formulate sodium alginate nanospheres of amphotericin B by controlled gellification method, evaluate its physicochemical characteristics such as particle size, drug loading capacity, in vitro release characteristics, and also the in vivo efficacy in candidiasis-induced animal models. An important requisite in the realization of an injectable nanoparticle delivery system is the proper choice of the polymeric substrate. The polymeric bulk must fulfil many requirements as well as have suitable mechanical properties and biodegradability. Therefore, a suitable polymer such as sodium alginate was selected for the study as it met these requirements.

\section{EXPERIMENTAL Materials}

Amphotericin B was generously donated by Synbiotics Ltd, Baroda, India. Other materials used include sodium alginate, calcium chloride, poly-l-lysine, dialysis bag (Sigma Inc USA). SEM (JSM-6400 scanning electron microscope, Tokyo, Japan), research centrifuge R24 (Remi instruments), UV spectrophotometer (UV 160 A Shimadzu, Japan) were the equipments used for the study.

\section{METHODS \\ Preparation of sodium alginate nanospheres by controlled gellification method}

Sodium alginate nanospheres were prepared by the method reported by Rajaonarivony et.al, ${ }^{10}$. $2 \mathrm{ml}$ of calcium chloride $(18 \mathrm{mM})$ was added to $38 \mathrm{ml}$ of sodium alginate solution $(0.1 \% \mathrm{w} / \mathrm{v})$ to induce gellification. Then $16 \mathrm{ml}$ of poly-l-lysine $(0.1 \% \mathrm{w} / \mathrm{v})$ was added to form a polyelectrolyte complex. The nanospheres suspension obtained was stirred for $2 \mathrm{~h}$ and kept overnight for stabilization. The nanospheres were separated by ultra centrifugation at $20000 \mathrm{rpm}$ for 45 minutes and dried under vacuum to form a flaky mass, which on redispersion in sterile water for injection, produced discrete particles.

\section{Determination of particle size}

The nanospheres were spread over a glass slide and dried under vacuum at room temperature $\left(25^{\circ} \mathrm{C}\right)$. The sample was shadowed in a cathodic evaporator with a gold layer $20 \mathrm{~nm}$ thick. The diameters of all the spheres in each field were calculated using a JSM-6400 scanning electron microscope (Tokyo, Japan). ${ }^{11}$

\section{Study on drug to polymer ratio}

To determine the drug: polymer ratio ${ }^{12}$, five batches of nanospheres containing various concentrations of drug were prepared. In each batch the concentration of drug was varied, while other processing variables were kept constant. 
Amphotericin B equivalent to $10 \mu \mathrm{g} / \mathrm{ml}$ was dissolved in $38 \mathrm{ml}$ of sodium alginate solution $(0.1 \%)$. Then $2 \mathrm{ml}$ of calcium chloride $(18 \mathrm{mM})$ and $16 \mathrm{ml}$ of poly-l-lysine $(0.1 \%)$ was added, stirred for $2 \mathrm{~h}$ and kept overnight. The resulting nanospheres were separated at 20000 rpm by ultracentrifugation. The nanospheres were dried under vacuum. This batch coded ASA-I. Similarly ASA-II, ASA-III, ASA-IV and ASA-V were prepared, containing $20 \mu \mathrm{g} / \mathrm{ml}, 30 \mu \mathrm{g} / \mathrm{ml}$, $40 \mu \mathrm{g} / \mathrm{ml}$ and $50 \mu \mathrm{g} / \mathrm{ml}$ of amphotericin B, respectively. The batches were subjected to particle size analysis by SEM.

\section{Determinations of drug content}

An ultracentrifugation technique ${ }^{13}$ was used to separate the free drug from nanospheres and to estimate the drug loading of the nanospheres. The final colloidal suspensions were ultra centrifuged at $10000 \mathrm{rpm}$ at $22 \pm 2^{\circ} \mathrm{C}$ for $0.5 \mathrm{~h}$. The supernatant was analyzed for drug content by measuring the absorbance at $345 \mathrm{~nm}$ using UV spectrophotometer.

In vitro release studies

The drug release assessment was carried out following the procedure reported earlier ${ }^{15}$, which employed a diffusion cell. A Sigma dialysis membrane was fixed to one end of a permeation cell. The nanospheres were placed in the cell donor compartment and the cell was immersed in a beaker containing $50 \mathrm{ml}$ of phosphate buffer (pH 7.4) which serve as a receptor compartment. The cell was immersed to a depth of $1 \mathrm{~cm}$ below the surface of the receptor solvent. The medium in the receptor compartment was agitated using a magnetic stirrer and temperature of $37 \pm 1^{\circ} \mathrm{C}$. $5 \mathrm{ml}$ of the sample from the receptor compartment was taken at various intervals of time over a period of $24 \mathrm{~h}$ and each time replaced with fresh buffer. The samples withdrawn were estimated spectrophotometrically at $345 \mathrm{~nm}$.

In vivo studies in candidiasis induced mice models

The institutional animal ethics committee of JSS College of pharmacy approved the protocol study. The in vivo study was carried out in candidiasis induced mice models as reported earlier ${ }^{14}$. A total of 30 animals were taken, and divided into 3 groups. All 3 groups were induced with candidiasis by intravenous administration of $0.1 \mathrm{ml}$ of $10^{5}$ to $10^{6}$ cells $/ \mathrm{ml}$ of cell culture suspension. After $24 \mathrm{~h}$ of candidiasis induction, the Group I was treated with nanospheres bound amphotericin B, Group II with free amphotericin $B$. In each case, bound drug and free drugs were dispersed in sterile water for injections. The dose of the drug was $100 \mathrm{mg} / \mathrm{kg}$ of animal weight and the volume of injection was $1 \mathrm{ml} / 100 \mathrm{~g}$ of mouse. The doses were given once a day for 7 consecutive days. Group III (control) was treated with solvent only. The animals were observed for mortality for 16 consecutive days. The mice were sacrificed at the end of $16^{\text {th }}$ day by cervical dislocation and dissected immediately. Organs like kidneys, lungs and liver were removed and homogenized in $5 \mathrm{ml}$ of sterile saline with Tween 80. The number of colony forming units (CFU) in each homogenate was determined by plate counts on Sabourands dextrose agar media (SDA) containing $0.05 \mathrm{mg} / \mathrm{ml}$ of chloramphenicol.

\section{RESULTS AND DISCUSSION}

Preparation of nanospheres by controlled gellification involves formation of calcium alginate complexes. The interaction between the calcium ions and the alginate polymer occurred at the level of the oligopolyglucuronic sequences. Furthermore, calcium ions induced a parallel packing of the oligopolyglucuronic sequences to give egg-box structures and the addition of poly-l-lysine allowed only strengthening of this system to obtain small and well defined particles. The obtained nanospheres were spherical and discrete with an average particle size of $419.6 \pm 0.28 \mathrm{~nm}$.

\section{Drug-loading capacity}

The drug carrier capacity of sodium alginate with respect to amphotericin B was determined assessing the drug: polymer ratio. As Table 1 shows, the drug loading capacity was observed to have a direct linear relationship, with the drug loading capacity increasing as the concentration of drug increased. Thus, it can be said that the saturation capacity of the polymer with respect to the selected drug occurred at a relatively lower concentration and at a faster rate. 
Release kinetic analysis

The in vitro study of all the five batches showed an initial burst release within 30 min and $60 \%$ of the drug was released in a slow manner over 24

h. In order to predict and correlate the release

Table 1: Amphotericin B - loading efficiency of sodium alginate nanospheres

\begin{tabular}{llc}
\hline Formulation code & $\begin{array}{c}\text { Drug concentration } \\
(\mu \mathrm{g} / \mathrm{ml})\end{array}$ & $\begin{array}{c}\text { Drug loading } \\
(\%)\end{array}$ \\
\hline ASA I & 10 & $10.7 \pm 0.2$ \\
ASA II & 20 & $13.5 \pm 0.6$ \\
ASA III & 30 & $17.2 \pm 0.8$ \\
ASA IV & 40 & $22.6 \pm 0.4$ \\
ASA V & 50 & $27.3 \pm 0.7$ \\
\hline
\end{tabular}

Table 2: In vitro release kinetics of amphotericin B- loaded nanospheres

\begin{tabular}{cccccc}
\hline $\begin{array}{c}\text { Formulation } \\
\text { code }\end{array}$ & $\begin{array}{c}\text { Drug } \\
\text { concentration } \\
(\boldsymbol{\mu g} / \mathbf{m l})\end{array}$ & $\mathbf{K \times 1 0 ^ { - 3 }}$ & $\mathbf{r}$ & $\mathbf{n}$ & $\mathbf{r}$ \\
\cline { 3 - 6 } & 10 & 2.6357 & 0.9951 & 0.5199 & 0.9447 \\
ASA I & 20 & 2.8368 & 0.9956 & 0.5132 & 0.9265 \\
ASA II & 30 & 2.8735 & 0.9708 & 0.5223 & 0.9131 \\
ASA III & 40 & 3.5046 & 0.9926 & 0.5437 & 0.9404 \\
ASA IV & 50 & 4.2236 & 0.9957 & 0.5583 & 0.9325 \\
ASA V & & & & &
\end{tabular}

Table 3: Percentage mortality and colony forming units of nanospheres bound amphotericin $B$ and free amphotericin $B$ in organs of RES.

\begin{tabular}{|c|c|c|c|c|}
\hline Drug treatment & \multirow[t]{2}{*}{ Mortality (\%) } & \multicolumn{3}{|c|}{ Number of colony forming units (CFU) } \\
\hline & & Kidney & Liver & Lungs \\
\hline $\begin{array}{l}\text { Amphotericin } \\
\text { free drug }\end{array}$ & 40 & $5 \times 10^{3}$ & $4.2 \times 10^{3}$ & $9.5 \times 10^{3}$ \\
\hline $\begin{array}{l}\text { Amphotericin } \\
\text { nanospheres } \\
\text { bound drug }\end{array}$ & 20 & $5.6 \times 10^{3}$ & $3.9 \times 10^{2 \star}$ & $4.5 \times 10^{2 *}$ \\
\hline Control & 100 & $1.8 \times 10^{4}$ & $7.5 \times 10^{3}$ & $1.9 \times 10^{4}$ \\
\hline
\end{tabular}




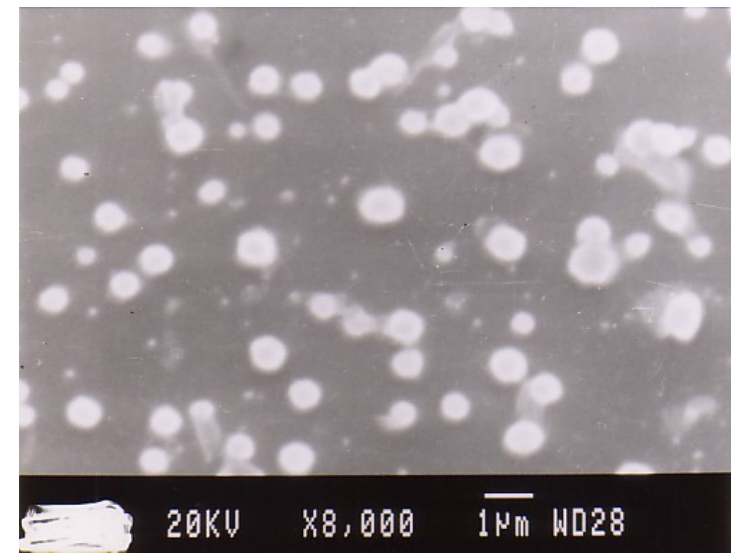

Fig 1: Scanning electron micrograph of sodium alginate nanospheres containing amphotericin B.

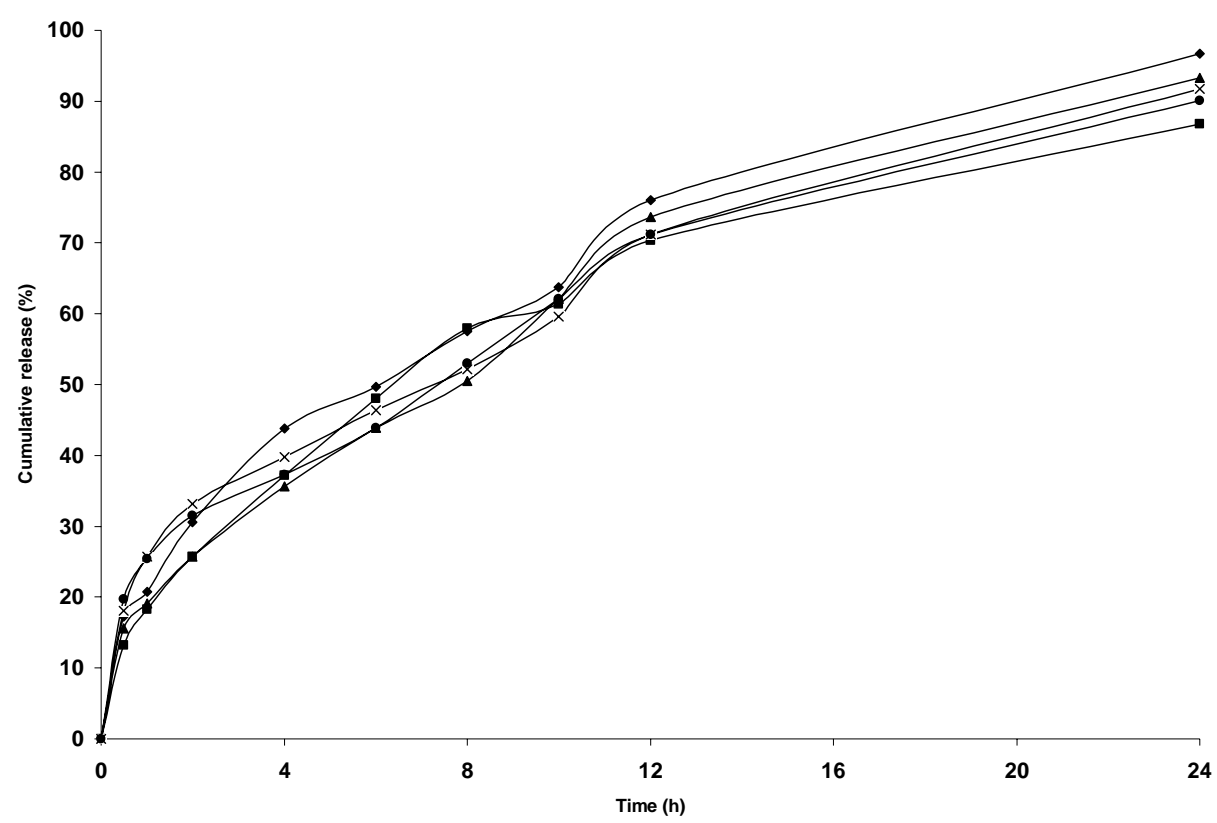

Fig 2: In vitro release profiles of amphotericin $B$ from different batches of drug loaded sodium

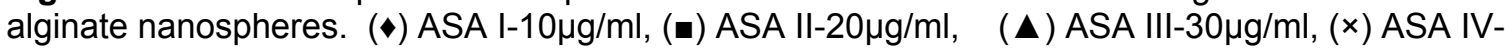
$40 \mu \mathrm{g} / \mathrm{ml}$ and $(\bullet)$ ASA- V-50 $\mu \mathrm{g} / \mathrm{ml}$. 
behavior of the drug from the hydrophilic matrix it is necessary to fit it to a suitable model. Hence, the release data were fitted according to the well known exponential equation ${ }^{16}$, often used to describe the drug release behavior from polymeric system:

$$
m t / m_{\infty}=k t^{n}
$$

Where $\mathrm{mt} / \mathrm{m}_{\infty}$ is the fractional release of the drug, ' $t$ ' is the release time, ' $k$ ' is a constant which indicates the properties of the macromolecular polymeric system and $n$ is the release exponent indicative of the mechanism of release. The ' $n$ ' value used for analysis of the drug release mechanism from the amphotericin $B$ nanospheres were determined from log $\left(\mathrm{mt} / \mathrm{m}_{\infty}\right)$ vs $\log (\mathrm{t})$ plots. To calculate the release constant, $k$, the logarithm of the remaining amphotericin B in nanospheres plotted versus time. The release of drug from nanospheres followed first order kinetics over a $24 \mathrm{~h}$ period. Drug loading is another factor that influenced the drug release rate from the nanospheres. Generally, increasing the drug content in the nanospheres increased. The values of ' $k$ ', ' $n$ ' and ' $r$ ' for five different batches are reported in Table 2 , and the ' $n$ ' value was within 0.5132 to 0.5583 . The results of kinetic analysis reveal that the release of amphotericin $B$ from sodium alginate nanospheres followed Fickian diffusion.

\section{In vivo studies}

An effective treatment of systemic candidiasis with amphotericin B requires comparatively higher concentrations of the antifungal drug in major target organs, such as the like lung and liver, which act as reservoirs of microbes and may lead to reoccurrence of infection. As Table 3 indicates, the nanosphere-bound drug is relatively more effective than the free drug against the candidiasis in terms of significant reduction in the colony forming units (CFU) in liver and lungs $(p<0.05$ by SNK test). The significant reduction in colony forming units (CFU) in the liver and lung could be to enhanced drug localization of nanosphere- bound drug as a result of macrophage uptake. The results obtained in this study showed a marked reduction in the CFU in organs of liver and lungs when compared with the CFU for groups treated with the free drug. The reduction in CFU as well as the fall in \% mortality may facilitate a reduction in the total dose required for therapy. This, in turn, means that dose-related systemic toxicities could also be reduced.

\section{CONCLUSION}

The formulated sodium alginate nanospheres of amphotericin B were found to be an effective and natural carrier in terms of discrete particle size, optimum drug loading capacity, satisfactory in vitro release characteristics, and in vivo antifungal activity for the passive delivery of amphotericin B. Furthermore, the nanospherebound drug may enhance drug localization in the RES. A possible beneficial fall-out is a reduction in total dose as well as dose-related systemic side effects.

\section{REFERENCES}

1. Fridkin SK, Jarvis WR and Garber, G. An overview of fungal infections. 1996, 1: 1-12.

2. Ellis $M$, Invasive fungal infections: evolving challenges for diagnosis and therapeutics. Molecular Immunology. 2002; 38: 947-957.

3. Rex JH, Walsh TJ and Sobel JD, Practice guidelines for the treatment of candidiasis, Clinical Infectious Diseases. 2000; 30: 662-678.

4. Warnock DW, Fungal infections in neutropenia: current problems and chemotherapeutic control. Journal of Antimicrobial Chemotherapy. 1998; 41: 95105.

5. Szoka FC, and Tang M, Amphotericin B formulated in liposomes and lipid based systems: a review. Journal of Liposome Research. 1996; 3: 363-375.

6. Mebela, TKM, Poupaert JH and Dumont P, Poly (diethyl methyl-lidene malonate) nanoparticles as primaquine delivery system to liver. Int. J. Pharm. 1992; 79: 29-38.

7. Fresta M, Pulgisi G, Giammona G, Cavallaro G, Micali $N$, and Furner $M$, Pefloxacin mesilate and ofloxacin loaded polyethyl cyanoacrylate nanoparticles: Characterization of the colloidal drug carrier formulation, J. Pharm. Sci. 1995; 84 : 895-901.

8. Irma AJM, Bakker - Woudenberg, Tenkate MT, Storm $G$, and Vanetten, EWM, Administration of liposomal agents and phagocytic function of the mononuclear phagocyte system, Int. J. Pharm. 1998; 162: 5-10,

9. Rodrigues JM, Fessi $H$, Bories C, Puisieux $F$ and Devissaguet J. Primaquine loaded poly (lactide) nanoparticles: Physiochemical study and acute tolerance in mice, Int. J. Pharm. 1995; 126: 253260.

10. Rajaonarivony $M$, Vauthier $C$, Couvrraze $G$, Puisieux $F$ and Couvreur $P$, Development of a new drug carrier made from alginate, J. Pharm. Sci. 1993; 82(9): 912. 
Sangeetha et al

11. Santhi K, Dhanaraj SA, Koshy M, Ponnusankar $S$ and Suresh B. Study of biodistribution of methotrexate loaded bovine serum albumin nanospheres in mice, Drug. Dev. Ind. Pharm. 2000; 26(12): 12931296.

12. Fawaz F, Guyot M, Lagueny AM and Devissaguet J.Ph. Ciprofloxacin loaded poly isobutyl cyano acrylate nanoparticles: Preparation and characterization, Int. J. Pharm. 1997; 158: 191-203.

13. Cavallaro G, Fresta M, Giammona G, Puglisi $G$ and Villari $A$. Entrapment of $\beta$-lactams antibiotics in poly ethyl cyano acrylate nanoparticles: Studies on the possible in vivo applications of this colloidal drug delivery system, Int. J. Pharm. 1994; 111: 31-41.
14. Suresh $B$, Kalyanaraman VR, Dhanasekaran $S$, Annadurai K, Dhanaraj SA and Balasubramanian $S$. Evaluation of santolina oil in search of new drugs against candidiasis, Indian Journal of Pharmacology. 1995; 27: 171.

15. Davison CJ, Smith KE, Hutchinson L, Mullane JE, Brookman JE, Patrak $K$ and Hardings SE. Physical and biological properties of water soluble polyelectrolyte complexes, Journal of Bioactive Compatable Polymer. 1990; 5: 267-282.

16. Korsmeyer RW, Gurny $R$, Doelkar $E$, Buri $P$ and Peppas NA. Mechanisms of solute release from porous hydrophilic polymers, Int. J. Pharm. 1983; 15: $23-25$. 